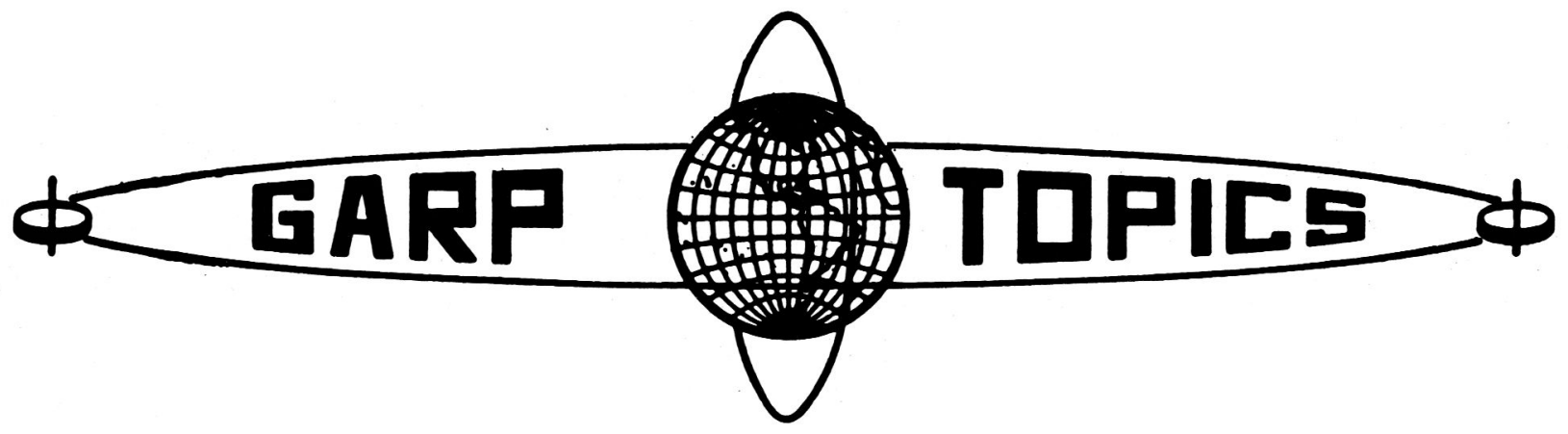

An occasional series reporting on U.S. and international GARP scientific, technical, and planning activities, developments, and programs, presented as a public service to the meteorological community by the American Meteorological Society through arrangements with the U.S. Committee on the Global Atmospheric Research Program of the National Academy of Sciences-National Research Council. Opinions expressed in "GARP Topics" do not necessarily reflect the point of view of the U.S. Committee.

\title{
A Note On Errors and Uncertainties in NCAR Aircraft Pyrgeometer Data from MONEX
}

\author{
Robert G. Ellingson \\ Department of Meteorology, University of Maryland, College Park, Md. 20742
}

\begin{abstract}
An analysis of the infrared irradiance data obtained from the upward- and downward-looking pyrgeometers on the NCAR Electra indicates that there are nonsystematic errors in the Monsoon Experiment (MONEX) data archived at the World Data Centers. The errors often exceed $8 \%$ of the downward flux and may be eliminated only by reanalyzing the $1 \mathrm{~Hz}$ data archived at NCAR. In addition, uncertainties in the measurement of the temperatures of the pyrgeometer surfaces lead to uncertainties in the corrected data, which may be important to particular applications.
\end{abstract}

\section{Introduction}

A major scientific goal of the summer Monsoon Experiment (MONEX) was to define the heat sources and sinks of the summer monsoon at the land and sea surface, within the at-

0003-0007/82/121387-03\$04.75

(c) 1982 American Meteorological Society mosphere, and at the top of the atmosphere (Fein and Kuettner, 1980). The primary data to be used for this specification consist of observations of the upward and downward long- and shortwave irradiances measured with aircraftmounted pyranometers (shortwave) and pyrgeometers (longwave). These data are also useful for identification of the radiative effects of atmospheric aerosols and clouds and for validation of techniques for calculating atmospheric radiation.

Reports on the accuracy of pyrgeometer observations are varied. Albrecht and Cox (1977) estimate the accuracy as 5\%, whereas MONEX literature lists a $1 \%$ accuracy specification (e.g., Bolhofer et al., 1981). Since the processed data were distributed to the World Data Centers, my research has discovered an error in the processing of pyrgeometer data obtained from NCAR aircraft through MONEX that often exceeds $5 \%$ of the flux. Furthermore, there are additional uncertainties in the reprocessed data that may be important for certain applications. In view of the possibility of improper interpretation of the currently distributed NCAR Electra pyrgeometer data, it is important that scientists using these data be informed of the uncertainties associated with them. 


\section{Basic equations}

The basic equation for determining the irradiance, $F$ (flux), incident on a pyrgeometer for the configuration used by NCAR is given as (Albrecht and Cox, 1977)

$$
F=\eta V+\epsilon \sigma T_{s}^{4}-k \sigma\left(T_{d}^{4}-T_{s}^{4}\right),
$$

where $\eta$ is the pyrgeometer responsivity, $V$ is the thermopile output voltage, $\epsilon$ is the emissivity of the blackened thermopile surface, $T_{s}$ is the absolute temperature of the thermopile surface (sink), $T_{d}$ is the temperature of the pyrgeometer dome, $\sigma$ is the Stephan-Boltzmann constant, and $k$ is an empirically determined constant. The first term in Eq. (1) represents the net radiation at the thermopile, the second term is the emission by the thermopile, and the third term accounts for the emission by the dome and internal reflections. This paper will be concerned with errors in the second and third terms of Eq. (1) only.

A comparison of a profile of pyrgeometer-observed fluxes with those that were calculated theoretically for a clear-sky MONEX observation period revealed some peculiarities, which I attributed initially to unseen cirrus, although similar effects were not seen on simultaneous shortwave radiation observations. Following an investigation it was discovered that the software used by the NCAR Research Aviation Facility (RAF) to determine the irradiance using Eq. (1) had the incorrect sign for the third term. Denoting the difference between the correct and incorrect flux as $\Delta F, \Delta F$ may be written as

$$
\Delta F=-2 k \sigma\left(T_{d}^{4}-T_{s}^{4}\right)
$$

Albrecht and Cox (1977) showed that the neglect of the dome sink temperature difference may result in errors in excess of $5 \%$ of the downward flux, depending upon the aircraft altitude and length of time at the flight level. Obviously, the NCAR aircraft pyrgeometer data may be corrected simply by adding $\Delta F$ to the recorded data.

Although NCAR employs precision thermistors and an excellent bridge circuit-measuring system theoretically capable of determining $T_{s}$ and $T_{d}$ to within a few hundredths of a degree, the calibrations conducted before and after MONEX showed a degradation of the thermistors over the period. Since the thermistor calibrations were not monitored during the course of the experiment, and since no catastrophic changes were detected in the data during the postexperiment data-processing, the data from each thermistor were reduced using the average of the two calibrations.

Assuming that the initial and final calibrations represent the temperature extremes for a given observation and assuming that random error is small, a given measurement allows a temperature $T$ and its uncertainty $\delta T$ to be written as

$$
T=\bar{T} \pm \delta T(T)
$$

where $\bar{T}$ is the temperature determined from a given voltage measurement using the average calibration and $\delta T$ is onehalf of the range between the values determined by the initial and final calibrations. Since the calibrations were not followed throughout the experiment, it is impossible to determine the sign of $\delta T$ for a given set of observations. The different uncertainties for the dome and sink temperatures, $\delta T_{d}$ and $\delta T_{s}$, respectively, lead to an uncertainty in the irradiance estimate, $\delta F$, which is given approximately as

$$
\delta F= \pm 4 \sigma \bar{T}_{s}^{3}\left(\epsilon \delta T_{s}+k\left(\delta T_{d}+\delta T_{s}\right)\right),
$$

where it has been assumed that $\delta T \ll T$. Note that $\delta F$ is independent of the number of observations.

\section{Calculations and results}

We have calculated values of $\delta T_{s}, \delta T_{d}$, and $\delta F$ for the pyrgeometers used on the NCAR Electra during MONEX using thermistor calibration and pyrgeometer empirical data provided by the NCAR RAF. The instrument responsivity, $\eta$, was determined by the Eppley Laboratory shortly before MONEX, and the values of $k$ were determined before the experiment following the laboratory procedure outlined by Albrecht and Cox (1977). As pointed out by Albrecht and Cox, the laboratory value of $k$ may differ from values determined from in-flight data. However, this analysis does not address the effect of that uncertainty. Table 1 lists values of $\delta T_{s}, \delta T_{d}$, and $\delta F$ for $\bar{T}_{s}=\bar{T}_{d}$ for each pyrgeometer for the range of temperatures experienced during the experiment. Note that the largest values of $\delta F$ and $\partial \delta F / \partial \bar{T}_{s}$ occur at high temperatures. As a result, the largest absolute uncertainties in the upward, downward, and net fluxes and in the net flux divergence due to the thermistor changes will occur at low altitudes. Note that $\delta F$ may be calculated for a particular set of flux measurements from Eq. (4), with $\delta T_{s}$ and $\delta T_{d}$ determined from linear interpolations of the values given in Table 1 to recorded values of $\bar{T}_{s}$ and $\bar{T}_{d}$, respectively.

As examples of the errors in the initial pyrgeometer data analysis and the uncertainties in the corrected data, Table 2 lists values of $\Delta F$ and $\delta F$ in comparison to the mean observed flux data and their standard deviations $\left(\sigma_{F}\right)$ for a vertical profile of fluxes obtained under clear-sky conditions over the Arabian Sea on 4 June 1979. The data shown are averages of $1 \mathrm{~Hz}$ data over the final two minutes of a five-minute, constant-level flight leg. The selection of these data generally insures that the sink and dome temperatures have reached their equilibrium values. The data show that the errors in the uncorrected downward flux data are larger than the uncertainty due to the thermistor calibration uncertainties. Values of $\Delta F$ amount to as much as $9 \%$ of the corrected downward flux, whereas $\delta F$ is the order of $5 \%$ of the downward flux. Note that $\sigma_{F}$ is two orders of magnitude smaller than $\Delta F$ or $\delta F$. It should be emphasized that the results shown in Table 2 are for clear-sky conditions with a nearly overhead sun. The magnitude of $\Delta F$ for the downward flux may be smaller when solar heating of the pyrgeometer is reduced, such as in overcast conditions or at night.

For the upward fluxes $\Delta F \leqslant \delta F$, and $\delta F$ amounts to approximately $5 \%$ of $F$ at low levels and decreases upward. If one were concerned only with the value of the upward flux but not with the vertical derivative, the correction of the upward flux data would not yield much added information. However, it should be noted that comparisons of the upward flux observations at low levels with calculations based on simultaneously observed sea surface temperatures from two different instruments indicate that there is a systematic error from an unidentified source of approximately $25 \mathrm{~W} \mathrm{~m}^{-2}$ in these data. 
TABLE 1. Temperature and flux uncertainties for the pyrgeometers on the NCAR Electra during MONEX. All temperatures are in degrees Celsius and $\delta F$ is in watts per square meter.

\begin{tabular}{rrrrrrr}
\hline \hline \multicolumn{4}{c}{ Upper pyrgeometer } & \multicolumn{4}{c}{ Lower pyrgeometer } \\
$T$ & $\delta T_{s}$ & $\delta T_{d}$ & $\delta F$ & $\delta T_{s}$ & $\delta T_{d}$ & \multicolumn{1}{c}{$\delta F$} \\
\hline-20.00 & 0.03 & 0.13 & 1.1 & 0.12 & 0.04 & 1.5 \\
-15.00 & 0.04 & 0.15 & 1.5 & 0.13 & 0.05 & 1.7 \\
-10.00 & 0.04 & 0.16 & 1.6 & 0.14 & 0.06 & 2.1 \\
-5.00 & 0.07 & 0.20 & 2.3 & 0.17 & 0.07 & 2.6 \\
0.00 & 0.11 & 0.28 & 3.7 & 0.20 & 0.10 & 3.3 \\
5.00 & 0.15 & 0.30 & 4.5 & 0.23 & 0.11 & 4.0 \\
10.00 & 0.23 & 0.37 & 6.5 & 0.32 & 0.13 & 5.6 \\
15.00 & 0.26 & 0.42 & 7.8 & 0.35 & 0.17 & 6.9 \\
20.00 & 0.28 & 0.43 & 8.7 & 0.49 & 0.19 & 9.6 \\
25.00 & 0.41 & 0.57 & 12.7 & 0.56 & 0.27 & 12.0 \\
30.00 & 0.48 & 0.74 & 16.5 & 0.66 & 0.40 & 15.9 \\
35.00 & 0.63 & 0.80 & 20.8 & 0.89 & 0.38 & 20.6 \\
40.00 & 0.69 & 0.82 & 23.2 & 0.91 & 0.35 & 21.7 \\
\hline
\end{tabular}

An examination of the differences between fluxes and uncertainties at successive levels shows somewhat different results. The average absolute percentage error of the upward flux differences of the uncorrected data is approximately $12 \%$ and the average percentage uncertainty due to the thermistor calibration changes is approximately $20 \%$. For the downward flux differences, the thermistor changes result in an average uncertainty of approximately $10 \%$. These differences are about an order of magnitude larger than the standard deviations of the observations.

Shown in Table 3 are radiative heating rates $(H)$, errors in the uncorrected heating rates $(\Delta H)$, the standard deviations of the mean heating rates $\left(\sigma_{H}\right)$, and uncertainties in the heating rates due to the thermistor uncertainties as calculated from the data shown in Table $2(\delta H)$. In this particular example the uncorrected data give essentially the same results as the corrected data in the lowest layer, but they overestimate the heating rate at higher levels. The large uncertainty of the heating rate due to thermistor uncertainties occurs because there is no information available concerning the signs of $\delta F$ for both the upward and downward fluxes on an individual flight. As a result, $\delta H$ may amount to $35 \%$ of the estimated heating rate. Again, the results may be different on other days and for different atmospheric layers.

\section{Summary and conclusions}

The purpose of this note is to alert the scientific community

TABLE 2. Observed fluxes and their uncertainties as a function of pressure $p$ for 4 June 1979. $F$ is the mean observed flux, $\sigma_{F}$ is the standard deviation of the mean, $\Delta F$ is the difference between the corrected and uncorrected mean fluxes, and $\delta F$ is the flux uncertainty due to the uncertainty of the dome and sink thermistor calibrations. Flux units are watts per square meter and pressure is in millibars.

\begin{tabular}{|c|c|c|c|c|c|c|c|c|}
\hline \multicolumn{5}{|c|}{ Downward } & \multicolumn{4}{|c|}{ Upward } \\
\hline$p$ & $F$ & $\sigma_{F}$ & $\Delta F$ & $\delta F$ & $F$ & $\sigma_{F}$ & $\Delta F$ & $\delta F$ \\
\hline 1006.5 & 424.8 & 0.52 & 29.8 & 20.9 & 452.8 & 0.21 & -4.3 & 23.7 \\
\hline 872.4 & 361.7 & 0.18 & 32.9 & 18.3 & 436.6 & 0.16 & -0.4 & 20.6 \\
\hline 748.7 & 295.9 & 0.09 & 24.3 & 11.4 & 409.4 & 0.07 & 2.6 & 14.0 \\
\hline 592.5 & 213.9 & 0.05 & 16.4 & 6.9 & 369.6 & 0.07 & 2.8 & 7.7 \\
\hline 503.3 & 178.2 & 0.05 & 12.2 & 4.7 & 355.9 & 0.05 & 4.2 & 4.9 \\
\hline
\end{tabular}

TABLE 3. Observed heating rates $\left({ }^{\circ} \mathrm{C} \mathrm{day}{ }^{-1}\right)$ and their uncertainties for 4 June 1979. $H$ is the heating rate for the layer determined from the mean corrected fluxes, $\sigma_{H}$ is the standard deviation of the mean heating rates, $\Delta H$ is the difference between heating rates calculated with the corrected and uncorrected flux data, and $\delta H$ is the uncertainty due to the thermistor uncertainties.

\begin{tabular}{rcccc}
\hline \hline Layer $(\mathrm{mb})$ & $H$ & $\sigma_{H}$ & $\Delta H$ & $\delta H$ \\
\hline $1006.5-872.4$ & -2.94 & 0.04 & -0.05 & 0.36 \\
$872.4-748.7$ & -2.63 & 0.02 & -0.79 & 0.92 \\
$748.7-592.5$ & -2.27 & 0.01 & -0.44 & 0.58 \\
$592.5-503.3$ & -2.08 & 0.01 & -0.53 & 0.47 \\
\hline
\end{tabular}

to the importance of making corrections to the NCAR aircraft pyrgeometer data processed through MONEX. The analysis indicates that if one is concerned with the upward longwave flux per se, correction of the upward fluxes will add little new information, but corrections of the clear-sky daytime downward fluxes are necessary in order to keep the uncertainty to within 5\%. The errors in the divergence of the uncorrected upward and downward fluxes are of the same magnitude as the uncertainties due to the thermistor calibration changes at all levels. In order to study the divergence of the upward and downward fluxes obtained during MONEX, it will be necessary to correct the data. Furthermore, use of the MONEX Electra data to determine radiative heating rates will require that particular attention be paid to correcting the pyrgeometer data and the estimation of the magnitude of the uncertainty due to the thermistor changes. However, it appears difficult to keep the uncertainty of the radiative heating rate under $10 \%$, at best.

Unfortunately, the data necessary to correct, and to estimate the uncertainty of, the MONEX pyrgeometer data are not included in the data currently archived at the World Data Centers. In order to correct the data, it is necessary to obtain a copy of the original $1 \mathrm{~Hz}$ data archived by RAF at NCAR.

Finally, in order to obtain better quality pyrgeometer data in future experiments, it will be necessary to monitor the changes of the thermistors mounted on the important instrument surfaces, along with the instrument responsivity. If this is not done the uncertainty of the observations will rival those of the much less expensive radiometersonde.

Acknowledgments. This research was sponsored in part by NSF under grant ATM 7821872, and computer time was furnished by the University of Maryland Computer Science Center. The author wishes to pay particular thanks to Richard Friessen and Keith Griffith of NCAR for their help in identifying the error in the pyrgeometer data and for providing the empirical data necessary for this research.

\section{References}

Albrecht, B., and S. K. Cox, 1977: Procedures for improving pyrgeometer performance. J. Appl. Meteorol., 16, 188-197.

Bolhofer, W., M. Chambers, D. Frey, J. Kuettner, and S. Unninayar, 1981: Summer MONEX U.S. research flight missions, May-July, 1979. $N C A R / T N-168+S+R$, National Center for Atmospheric Research, Boulder, Colo., 220 pp.

Fein, J. S., and J. P. Kuettner, 1980: Report on the summer MONEX field phase. Bull. Am. Meteorol. Soc., 61, 461-474. 